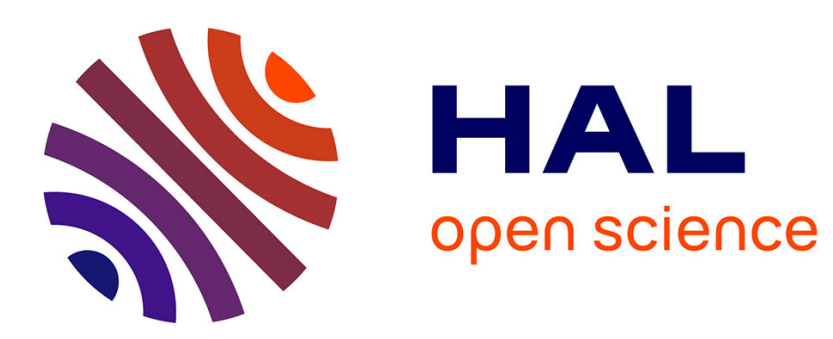

\title{
Scaling behavior of particle breakage in granular flows inside rotating drums
}

Luisa Orozco, Jean-Yves Delenne, Philippe Sornay, Farhang Radjai

\section{To cite this version:}

Luisa Orozco, Jean-Yves Delenne, Philippe Sornay, Farhang Radjai. Scaling behavior of particle breakage in granular flows inside rotating drums. Physical Review E , 2020, E 101, pp.052904. 10.1103/PhysRevE.101.052904 . hal-02569714

\section{HAL Id: hal-02569714 \\ https://hal.science/hal-02569714}

Submitted on 11 May 2020

HAL is a multi-disciplinary open access archive for the deposit and dissemination of scientific research documents, whether they are published or not. The documents may come from teaching and research institutions in France or abroad, or from public or private research centers.
L'archive ouverte pluridisciplinaire HAL, est destinée au dépôt et à la diffusion de documents scientifiques de niveau recherche, publiés ou non, émanant des établissements d'enseignement et de recherche français ou étrangers, des laboratoires publics ou privés. 


\title{
Scaling behavior of particle breakage in granular flows inside rotating drums
}

\author{
Luisa Fernanda Orozco, ${ }^{1,2, *}$ Jean-Yves Delenne, ${ }^{3}$ Philippe Sornay, ${ }^{1}$ and Farhang Radjai ${ }^{2}$ \\ ${ }^{1}$ CEA, DEN, DEC, SA3E, LCU, 13108 Saint Paul les Durance, France \\ ${ }^{2}$ LMGC, CNRS, University of Montpellier, France \\ ${ }^{3}$ IATE, INRA, CIRAD, Montpellier SupAgro, University of Montpellier, France
}

\begin{abstract}
We perform systematic particle dynamics simulations of granular flows composed of breakable particles in a $2 \mathrm{D}$ rotating drum to investigate the evolution of the mean particle size and specific surface as a function of system parameters such as drum size, rotation speed, filling degree, and particle shape and size. The specific surface increases at a nearly constant rate up to a point where particle breakage begins to slow down. The rates of particle breakage for all values of system parameters are found to collapse on a master curve when the times are scaled by the characteristic time defined in the linear regime. We determine the characteristic time as a function of all system parameters, and we show that the rate of particle breakage can be expressed as a linear function of a general scaling parameter that incorporates all our system parameters. This scaling behavior provides a general framework for the upscaling of drum grinding process from laboratory to industrial scale.
\end{abstract}

Keywords: Granular materials, Upscaling, particle breakage, Grinding, Rotating drum, Discrete Element Method, Bonded-Cell method, Contact Dynamics Method

\section{INTRODUCTION}

Rotating drums or tumbling mills are used in numerous industrial applications for grinding, mixing and particle agglomeration [1-6]. The system consists of a hollow drum subjected to a rotation velocity around its principal axis. In the grinding process, particle breakage occurs as a result of frictional and collisional interactions between particles and with the drum walls. Since most of these devices operate in the rapid-flow regime, i.e. the so-called cascading or cataracting regimes, the granular flow has an inertial nature and develops a complex geometry with inhomogeneous flow patterns and curved free surface. For this reason, the grinding mechanisms in rotating drums are complex and poorly understood from physical and mechanical points of view, and their scaling with operating parameters is an open issue.

Previous studies of the grinding process in rotating drums have been performed by means of experimental measurements, numerical simulations, and mechanistic or stochastic models. Experimentally, the test conditions impede a continuous track of particle breakage. Some properties such as particle size distribution are therefore measured from samples taken at different instants of the test [7-9]. The numerical simulations have the advantage of allowing for different particle shapes to be considered and continuous track of the particles and their mechanical interactions [10, 11], but the existing particle dynamics methods that take into account particle breakage require high numerical performance, and the computational limitation in the number of particles impedes statistical representativity of the samples [12-14]. Finally, the population balance models (PBM) combine particle breakage probability, usually obtained from single impact tests, with a mass transfer function to sequentially predict the evolution of particle size distribution during grinding $[15,16]$. One disadvantage of this method is the large number of parameters

\footnotetext{
*luisa.orozco@umontpellier.fr
}

that must be tuned for each specific case. In most cases, the calibration is based on experimental results, and some functions such as the mass transfer are fully empirical as their measurement in experiments is not possible. Furthermore, these models do not directly account for the mechanics of particle fracture in multicontact configurations, in which the breakage mechanisms are substantially different from those in a single particle impact test.

As an example of recent work in this field, it is worth mentioning models that combine the Discrete Element Method (DEM) for particle interactions and PBM for predicting the size distribution of the fragments [17, 18]. Cleary et al. [19] performed numerical simulations of a semi-autogenous mill in which the particles that reach a given small size can escape through slots of the drum wall located next to the lifters. In this work, the condition for particle breakage and the generated fragments were determined from the incremental breakage theory proposed by Vogel and Peukert [20]. The breakage probability was thus determined by a Weibull probability function whose parameters were fitted to the data of single particle impact tests. This survival probability function has been used by several authors to determine the evolution of particle distribution [21-23]. Despite their genuine character, it is generally difficult to evaluate the success of such models and their calibration by direct comparison with the real mechanisms that lead to particle breakage in dense granular flows.

In practice, we believe that a realistic model of the grinding process based on multicontact interactions should make it possible to reach an upscaling model for rotating drums, as a challenging problem for industrial purposes. Nearly all attempts to find relationships for the upscaling of drum size are either based on a dimensional analysis of the relevant physical quantities [24] or on the performance of the processes that take place inside rotating drums [25]. However, a fully predictive model should be able to discern the generic and specific features of the grinding process and to link the system parameters with the overall performance.

In this paper, we use numerical simulations to investigate 
the effect of system parameters on the grinding process in a $2 \mathrm{D}$ rotating drum. We rely on the contact dynamics method as a DEM algorithm and the discretization of the particles into bonded polygonal cells that an break apart, known as Bonded Cell Method (BCM) [10, 26-30]. The particles can thus break into fragments of different sizes down to the smallest cell size. We vary system parameters such as drum size, rotation speed and filling degree, to quantify the effect of each parameter on the granular flow and evolution of grinding in terms of the mean particle size and specific surface of the material. The specific surface is defined as the sum of the surface areas (the perimeter in 2D) of all particles divided by their total weight. We also consider the spatial map of the breakage events in correlation with drum flow parameters. Then, we examine the possibility of expressing the rate of particle breakage in terms of a single scaling parameter accounting for all system parameters. As we shall see, the results can be interpreted in terms of a characteristic time with a nontrivial dependence on system parameters.

In the following, we first introduce the methodology and simulation parameters in Secs. II A and II B. In Sec. III, we present the evolution of particle size distribution and specific surface as a function of rotation speed, filling degree, and drum size, and their scaling with a characteristic time. Then, in Sec. IV, we derive an expression for this time and breakage rate in terms of system parameters. We will conclude with a discussion of the results and outlooks of this work.

\section{NUMERICAL PROCEDURES}

\section{A. Contact Dynamics method and BCM}

The numerical simulations were performed by means of the contact dynamics method together with the Bonded-cell method (BCM) for particle breakage. Contact dynamics is a discrete-element method in which perfectly rigid particles interact through frictional contacts, and the particle motions are calculated by a step-wise implicit scheme [31-33]. In contrast to soft-particle DEM, the nonlocal strains are defined from particle overlaps, and the velocities and contact forces are calculated at the same time by an iterative process accounting for the contacts as unilateral constraints. As the particle overlaps do not need to be resolved, the time step can be much larger than in soft-particle DEM but a large number of iterations are required at each time step to converge to a solution for velocities and forces. The particle positions and contact network are then updated from the calculated velocities.

In the BCM, each particle is subdivided into smaller independent primary elements or cells by means of a Voronoï tessellation, and thus the particle volume is exactly equal to the sum of cell volumes [26, 27, 29, 30, 34]. During the generation process, the average cell size $d_{\text {cell }}$ is fixed, but the cell shapes are random. For the sake of geometric consistency, we choose also polygonal particle shapes as shown in Fig. 1. We used regular polygon-shaped particles of different nmber of sides $\left(n_{\text {sides }}\right)$ such as pentagons $\left(n_{\text {sides }}=5\right)$, hexagons

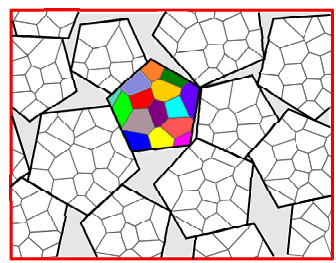

(a)

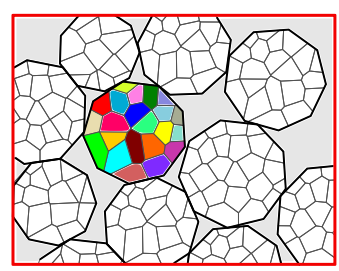

(c)

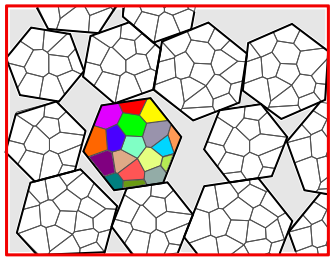

(b)

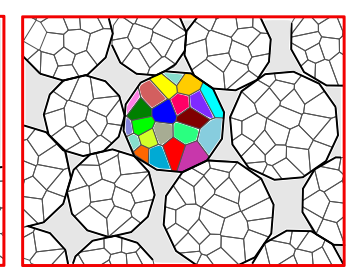

(d)
FIG. 1. Voronoï tessellation applied to (a) pentagonal particles $\left(n_{\text {sides }}=5\right)$, (b) hexagonal particles $\left(n_{\text {sides }}=6\right)$, (c) nonagonal particles $\left(n_{\text {sides }}=9\right)$, $(\mathrm{d})$ dodecagonal particles $\left(n_{\text {sides }}=12\right)$. The cells are represented by different arbitrary colors.

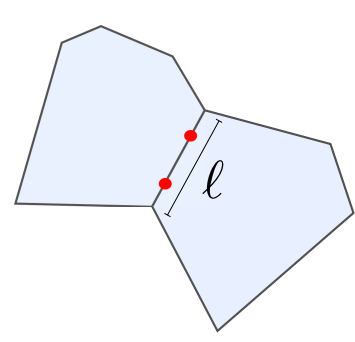

(a)

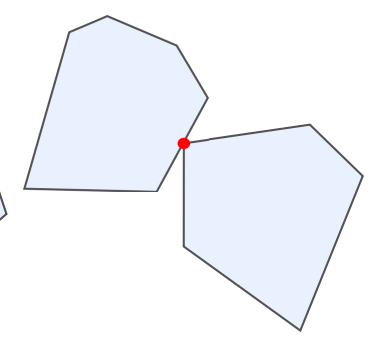

(b)
FIG. 2. (a) Side-side double-bond contact, (b) vertex-side single-bond contact.

$\left(n_{\text {sides }}=6\right)$, heptagons $\left(n_{\text {sides }}=7\right)$, nonagons $\left(n_{\text {sides }}=9\right)$ and dodecagons $\left(n_{\text {sides }}=12\right)$.

As the drum rotates, different types of contact can arise between particles: side-side, vertex-side, and vertex-vertex, as displayed in Fig. 2. The geometrical detection and representation of these contacts involves the definition of a common line for each pair of particles whose orientation determines the contact reference frame (normal and tangential directions). To each side-side contact we attribute two bond points located on the common line that can be projected onto each of the sides in contact. The total contact force at a side-side interface is therefore the sum of the forces acting at the two bonds attributed to the contact. At the vertex-side contacts a common line is also defined, but the contact involves a single bond [32].

By construction, in the initial configuration all the cell-cell interfaces belonging to each particle are side-side contacts, and each interface is represented by two cohesive bonds. The breakage requires in the first place a mechanical condition expressed as a stress threshold representing the material strength above which a crack can be initiated. But, 


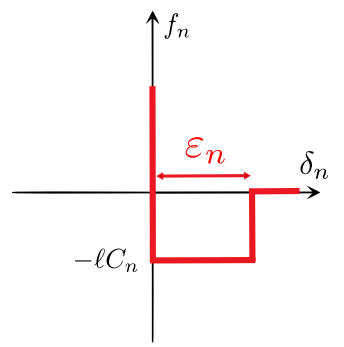

(a)

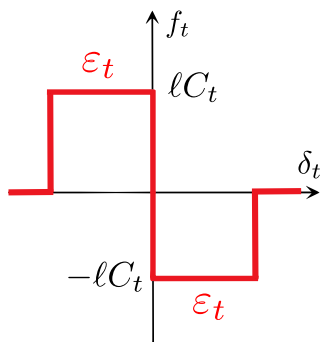

(b)

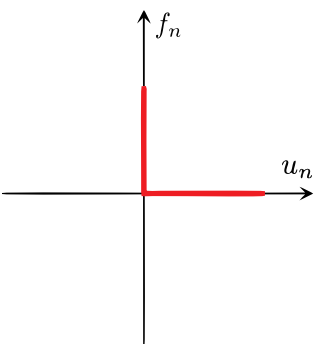

(a)

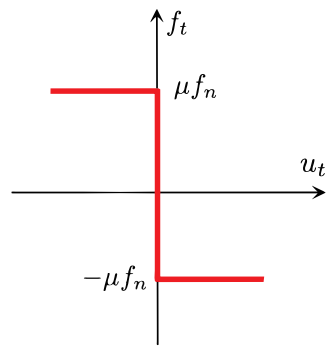

(b)
FIG. 3. Behavior of a cohesive contact between cells (a) in the normal direction, (b) in the tangential direction. $\ell$ is the side length, and $\delta_{n}$ and $\delta_{t}$ denote the local displacements between the two cells in the normal and tangential directions, respectively.

thermodynamically, a crack will not propagate unless an amount of mechanical energy, corresponding to the fracture energy or the toughness of the material is supplied. Hence, we assume that the fracture of a bond is controlled by two criteria [29]. The first criterion is a tensile stress threshold $C_{n}$ in the normal direction and a shear stress threshold $C_{t}$ in the tangential direction. In either direction, below the corresponding stress threshold, which represents the internal cohesion or strength of the particle, the relative movement of the two cells is forbidden. The second criterion is a fracture energy $\mathcal{W}$ that must be consumed by the relative displacements at the stress threshold as in the classical fracture mechanics. This energy criterion implies that the normal separation $\varepsilon_{n}$ at a bond should reach a threshold given by

$$
\varepsilon_{n}=\frac{W}{\ell C_{n}}
$$

where $\ell$ is the area (length in 2D) of the interface. At this distance the bond breaks, and a surface (length in 2D) equal to $\ell$ is created. In a similar vein, a bond can break when the tangential displacement reaches the critical distance

$$
\varepsilon_{t}=\frac{W}{\ell C_{t}}
$$

We assume that an inter-cell double-bond interface breaks as a whole if only one of its two bonds breaks following the two above criteria.

A graphical representation of this breakable interface law is shown in Fig. 3 as a relation between normal force and local displacement or gap $\delta$ between cells. Once a cohesive interface breaks, it turns into a frictional contact governed by the relation represented in Fig. 4. The same contact law governs also the vertex-side and vertex-vertex contacts, as well as the interactions with the walls. All the collisions are assumed to be perfectly inelastic. Further details about the implementation of the Bonded-cell method in the framework of Contact Dynamics can be found in [29].
FIG. 4. Behavior of a purely frictional contact (a) Signorini relation in the normal direction, (b) Coulomb friction law in the tangential direction. $u_{n}$ and $u_{t}$ denote the contact relative velocities in the normal and tangential directions, respectively.

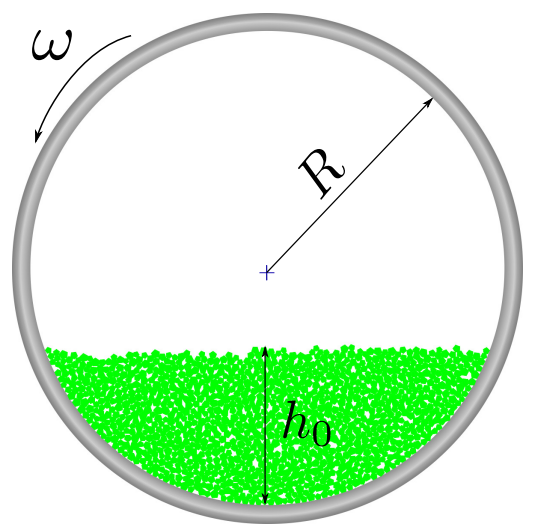

FIG. 5. Geometrical, mechanical and kinematic parameters of the simulated drums.

\section{B. Sample generation and test preparation}

We consider a smooth drum such that the particles inside the drum interact only through frictional contacts with the drum walls. No grinding media are added (as in ball mills), and the particle breakage is a consequence of granular flow (self-grinding as in autogenous mills). The initial particle diameters are distributed in a range between $d_{0}^{\min }$ and $d_{0}^{\max }$ with a uniform distribution of their volumes. Among all possible particle size distributions for a given size span (ratio $d_{0}^{\max } / d_{0}^{\min }$ ), this distribution yields one of the highest packing fractions $[35,36]$. Different particle shapes were also considered, as shown in Fig. 1. The cells belonging to each particle were generated such that the average size of cells $d_{\text {cell }}$ is proportional to particle size with around 20 cells per particle. The values of all parameters considered in this work are presented in Table I.

The generated sample is deposited inside a hollow drum (ring in 2D) of internal radius $R$ under the action of gravity $g$; See Fig. 5. Once all particles reach a state of force balance, a constant angular velocity $\omega$ is applied to the drum. Since the system is constantly evolving as a result of particle breakage, a steady state cannot be fully reached; See Fig. 6. However, a nearly steady flow state is reached after $\simeq 3$ rotations. The simulation is stopped when most particles are broken down 
TABLE I. Simulation parameters

\begin{tabular}{|c|c|c|c|}
\hline Parameter & Symbol & Value & Unit \\
\hline \multicolumn{4}{|l|}{ Geometrical parameters } \\
\hline Number of particles & $N_{p}$ & {$[147 ; 2350]$} & \\
\hline Number of cells per particle & $n_{\text {cells }}$ & {$[16 ; 36]$} & \\
\hline Number of cells (total) & $N_{\text {cells }}$ & {$[2008 ; 33574]$} & \\
\hline Particle density & $\rho$ & 2030 & $\mathrm{~kg} \mathrm{~m}^{-3}$ \\
\hline Drum internal radius & $R$ & {$[0.05 ; 0.2]$} & $\mathrm{m}$ \\
\hline Initial mean particle diameter & $\left\langle d_{0}\right\rangle$ & 2.5 or 6 & $\mathrm{~mm}$ \\
\hline Cell size & $d_{\text {cell }}$ & 0.5 or $1.2 \cdot 10^{-3}$ & $\mathrm{~mm}$ \\
\hline Filling degree & $f=h_{0} / R$ & {$[0.2 ; 0.5]$} & \\
\hline \multicolumn{4}{|l|}{ Mechanical parameters } \\
\hline Friction coefficient & $\mu$ & 0.4 & \\
\hline Normal restitution coefficient & $e_{n}$ & 0 & \\
\hline Tangential restitution coefficient & $e_{t}$ & 0 & \\
\hline Normal stress threshold & $C_{n}$ & 1 & $\mathrm{MPa}$ \\
\hline Tangential stress threshold & $C_{t}$ & 1 & $\mathrm{MPa}$ \\
\hline Critical normal distance & $\varepsilon_{n}$ & $5 \cdot 10^{-5}$ & $\mathrm{~m}$ \\
\hline Critical tangential distance & $\varepsilon_{t}$ & $5 \cdot 10^{-5}$ & $\mathrm{~m}$ \\
\hline \multicolumn{4}{|l|}{ Kinematic parameters } \\
\hline Rotation speed & $\omega$ & {$[1.57 ; 10.47]$} & $\mathrm{rad} / \mathrm{s}$ \\
\hline Froude number & $\mathrm{Fr}$ & {$[0.019 ; 0.838]$} & \\
\hline Time step & $\delta t$ & $1 \cdot 10^{-5}$ & $\mathrm{~s}$ \\
\hline Gravity acceleration & $g$ & 9.81 & $\mathrm{~m} / \mathrm{s}^{2}$ \\
\hline
\end{tabular}

to the smallest possible fragment size $d_{\text {cell }}$. This condition can also be identified by following the mean particle size and specific surface, which evolves with time and level off after a number of drum rotations. For the value of strength threshold $C_{n}$ used in the simulations, the total simulated physical time for reaching significant particle breakage is of the order of 60 seconds, requiring most of time long-run simulations. It should be noted that self-grinding occurs when the particle strength is low-enough to allow for particle breakage in the cascading regime as a result of particle weights or collisional energies. The scaling that will be derived in this paper from the simulations quantifies the effect of particle strength on the breakage rate.

Since this work is focused on the effect of system parameters on the grinding process, we performed several sets of simulations, each time changing one parameter while keeping constant values for other parameters. In a set of runs, the drum radius was fixed to $R=0.075 \mathrm{~m}$, for different rotation speeds $\omega \in[3.14,5.24,7.33,10.47] \mathrm{rad} / \mathrm{s}$ and filling degrees $f \in[0.2,0.25,0.3,0.35,0.4,0.45,0.5]$. The filling degree is the ratio of the filling height measured in the initial state $\left(h_{0}\right)$ to the drum radius, as shown in Fig. 5. In these simulations, pentagonal particles $\left(n_{\text {sides }}=5\right)$ was considered). We also simulated systems with polygonal particle shapes with different numbers of their sides $n_{\text {sides }}$, which is a measure of their roundness. In this set of simulations, the mean particle size was $2.5 \mathrm{~mm}$.

In another set of runs, drums of different sizes $R / r \in$ $[16,25,33,50,66]$ were simulated. For these simulations,
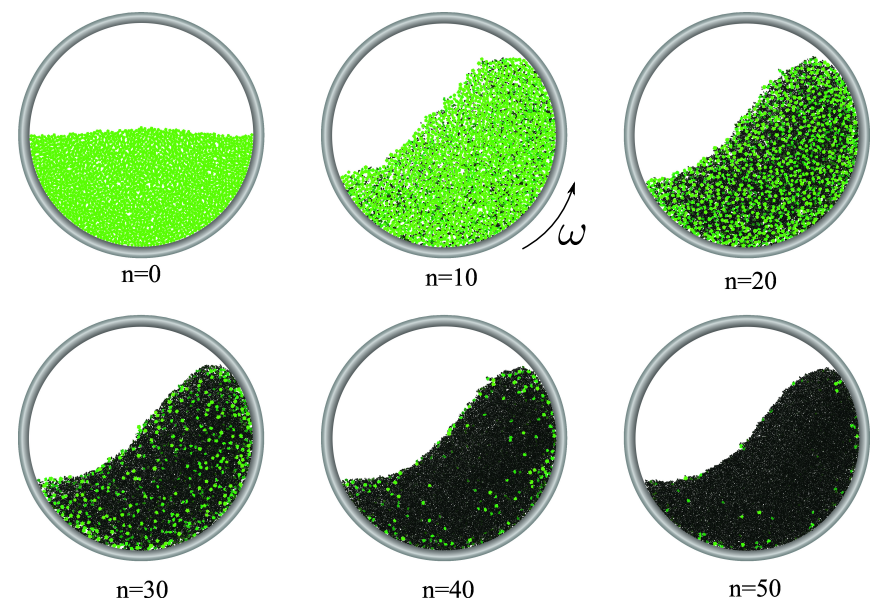

FIG. 6. Snapshots of a rotating drum simulation for different numbers of rotations $n$ for $\omega=5.24 \mathrm{rad} / \mathrm{s}$. The color is proportional to the damage, defined by the number of cells detached from a particle, represented on color scale from bright green for intact particles to black for highly-damaged particles.

particles of a larger mean size $d_{0}=6 \mathrm{~mm}$, but with the same size distribution and shape as in the first set were used. Consistently, the cell size was also modified to $\left\langle d_{\text {cell }}\right\rangle=1.2$ $\mathrm{mm}$ so that the number of cells per particle remains equal to 20. The largest drum was filled with 2350 particles, corresponding to a total number of 33574 cells. Finally, we also performed a set of simulations with constant values of the Froude number

$$
\operatorname{Fr}=\frac{R \omega^{2}}{g}
$$

by changing both the rotation speed $\omega$ and drum size $R$.

\section{EFFECTS OF SYSTEM PARAMETERS ON PARTICLE BREAKAGE}

\section{A. Rotation speed}

We analyze here the simulations with varying rotation speeds $\omega$, which is the most influential parameter on the flow regime [37]. Figure 7 shows the flow patterns in a drum with increasing rotation speed but the same size of $R=0.075 \mathrm{~m}$ and filling degree $f=0.51$. We see that by increasing $\omega$ from 1.57 to $12 \mathrm{rad} / \mathrm{s}$, the granular material flows successively in the rolling $(\omega=1.57$ and $3.14 \mathrm{rad} / \mathrm{s})$, cascading ( $\omega=5.24$ and $7.85 \mathrm{rad} / \mathrm{s})$, cataracting ( $\omega=10.47$ $\mathrm{rad} / \mathrm{s})$, and centrifuging regimes ( $\omega=12 \mathrm{rad} / \mathrm{s})$. The average free surface profiles in this set of simulations are shown in Fig. 8. The evolution of the free surface from a nearly flat surface flow to a curved S-shape indicates a transition from the rolling regime to the cascading regime. In the rolling regime, the centrifugal forces at the drum surface are low compared to the inertial forces inside the flow, and therefore the particles roll down the slope as a flat free surface granular flow. In the cascading and cataracting regimes the centrifugal 

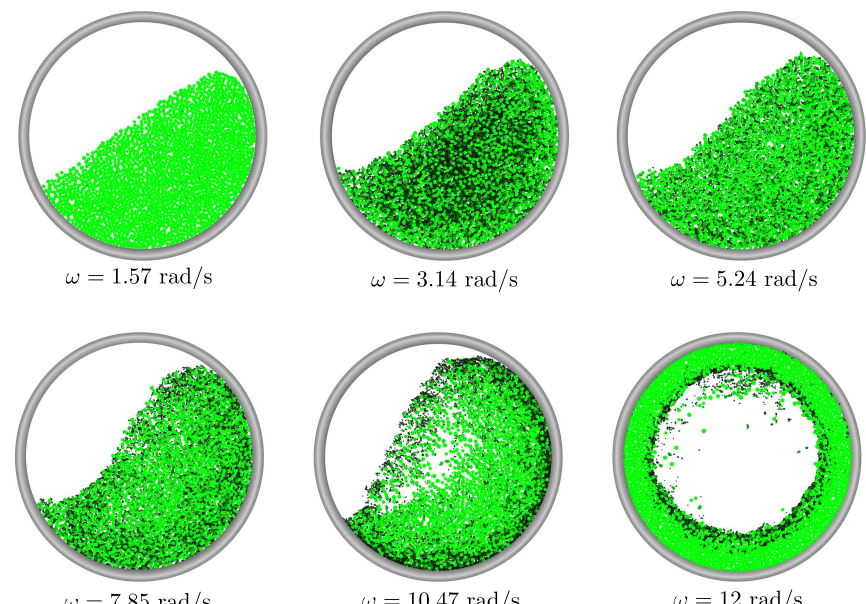

FIG. 7. Flow regimes displayed after 13.75 rotations, for $R=0.075$ $\mathrm{m}, f=0.51$, and increasing rotation velocity $\omega$. By increasing $\omega$, the Froude number varies from 0.02 to 1 . The color is proportional to particle damage, from bright green for intact particles to black for highly-damaged fragments.

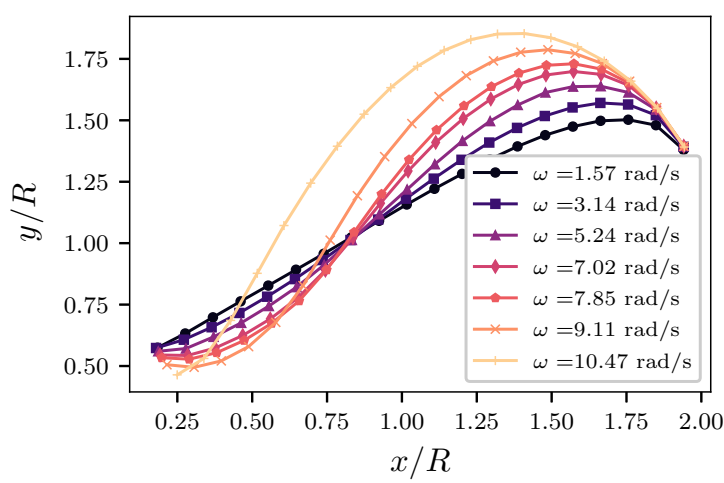

FIG. 8. Free surface profiles for different values of $\omega$.

forces become more important. In these regimes, the particles reach higher positions from which they feed a S-shape free surface in the case of a cascading flow or are thrown into the granular bed along ballistic trajectories in the cataracting regime. In the centrifugal regime the centrifugal forces prevail and consequently the particles stick to the walls. The amount of damaged particles for the same number of drum rotations is not the same in different regimes. In the following, we consider only the rolling and cascading regimes, as well as the beginning of the cataracting regime for the analysis of particle breakage.

Figure 9(a) shows the evolution of the mean particle diameter $\langle d\rangle$ as a function of time for different values of $\omega$. The diameters are normalized by their initial mean value $\left\langle d_{0}\right\rangle$. The mean size begins to decrease slowly, but at an increasing rate. At some point in time when the rate reaches its maximum value, which depends on $\omega$, the size reduction continues at decreasing rate until the minimum fragment size, i.e. the mean cell size $\left\langle d_{\text {cell }}\right\rangle$, is reached.

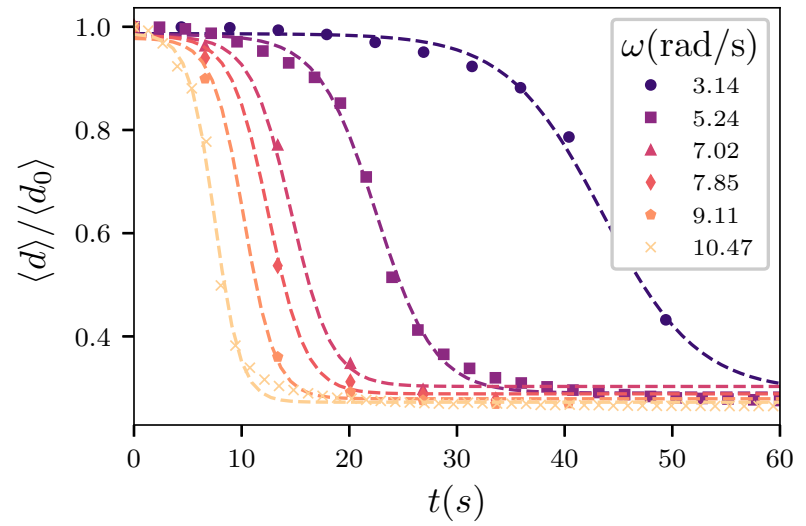

(a)

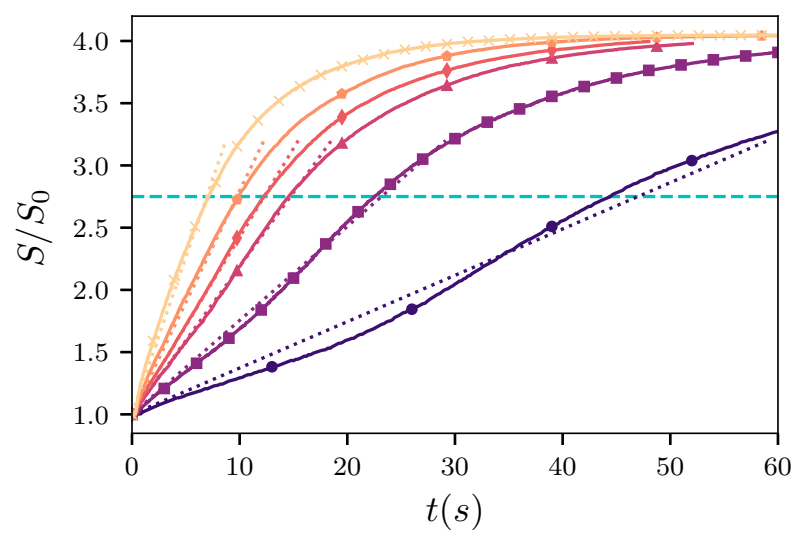

(b)

FIG. 9. (a) Evolution of the mean particle size $\langle d\rangle$ normalized by the initial mean particle size $\left\langle d_{0}\right\rangle$ for different values of the rotation speed $\omega$. The dashed lines correspond to a tangent hyperbolic form $\langle d\rangle /\left\langle d_{0}\right\rangle \sim \tanh (t)$. (b) Evolution of the specific surface $S$ normalized by the initial specific surface $S_{0}$. The dashed lines are linear fits up to a transition point to nonlinear regime.

This slowdown reflects the decreasing number of breakable fragments in the system. The evolution of the specific surface $S$ normalized by its initial value $S_{0}$ is shown in Fig. 9(b). Its maximum value is 4 , corresponding to the limit where all particles are fully broken into fragments of cell size. The initial evolution is nearly linear with a slight waviness in all cases but more pronounced for small values of $\omega$ where the evolution is slower. This feature reflects the initial adjustment of the granular flow to drum rotation since the rotation speed is applied instantaneously to an initially static bed. The evolution of the specific surface slows down after the transition point.

In the linear regime, the effect of $\omega$ and other system parameters can be quantified by using the average grinding rate $\dot{S} / S_{0}$. Figure 10 (a) shows $\dot{S} / S_{0}$ as a function of $\omega$. The rate increases as $\omega^{3 / 2}$. This means that the effect of rotation rate is not simply a change of timescale, in which 


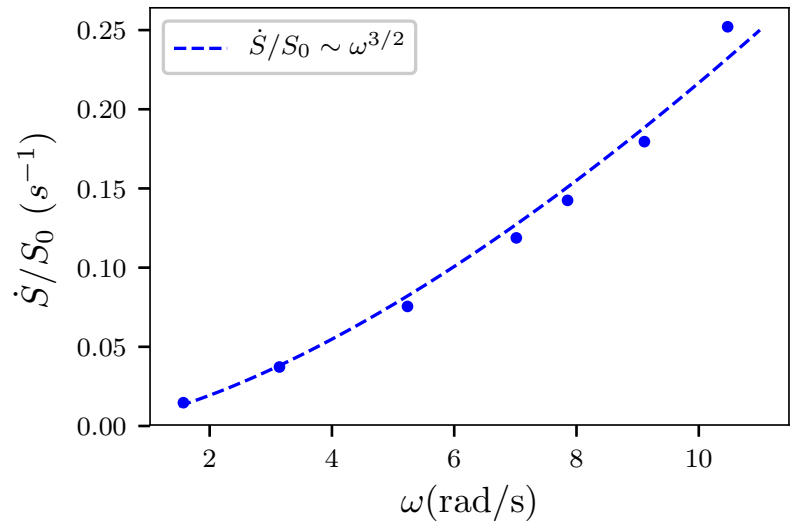

(a)

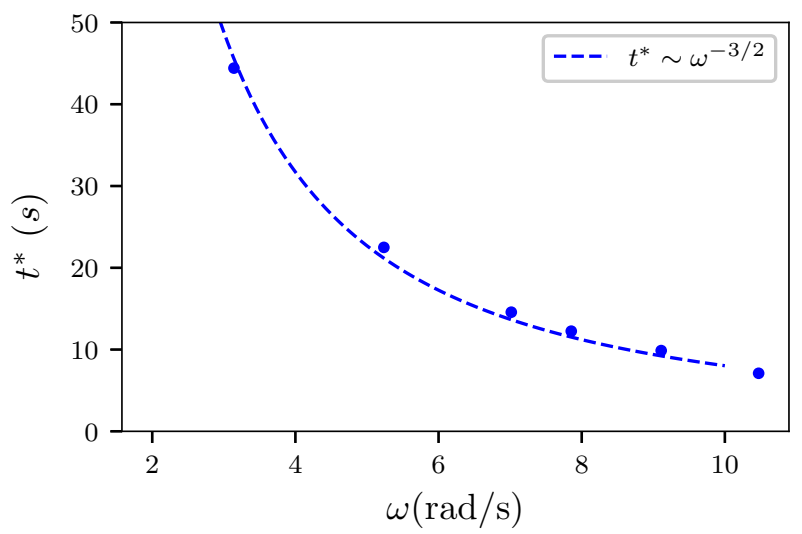

(b)

FIG. 10. (a) Rate of increase of specific surface [dashed lines in Fig. 9(b)]; (b) Characteristic time $t^{*}$ as a function of rotation speed $\omega$.

case the grinding rate would simply increase proportionally to $\omega$. However, the shapes of the evolution curves suggest that the transition to the nonlinear regime occurs always for $S / S_{0} \simeq 2.75$. This value is nearly half of the maximum specific surface that can be generated in our system. A characteristic time $t^{*}$ can thus be defined by

$$
S\left(t^{*}\right)=2.75 S_{0} .
$$

Figure 10(b) shows that this characteristic time varies as $\omega^{-3 / 2}$. Hence, we expect that all the data will collapse on a single curve when normalizing time by $t^{*}$. The mean particle size and specific surface are displayed in Fig. 11 as a function of normalized time. We see that, up to the aforementioned small waviness, we obtain a nice collapse of the data for both $\langle d\rangle$ and $S$.

Figure 12 shows the maps of the local densities of breakage events for three values of $\omega$. These maps were built by tracking the position of each bond prior to its breakage. The density at each point represents the probability of breakage at that point. In general, it is assumed that breakage events take

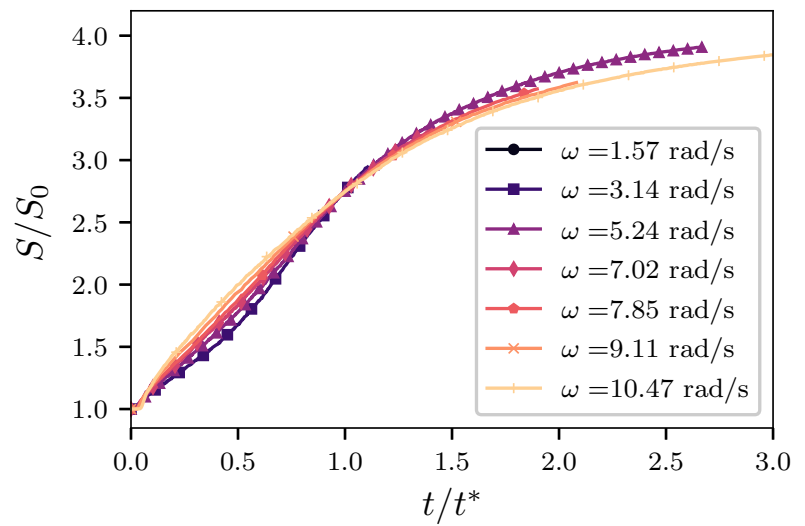

(a)

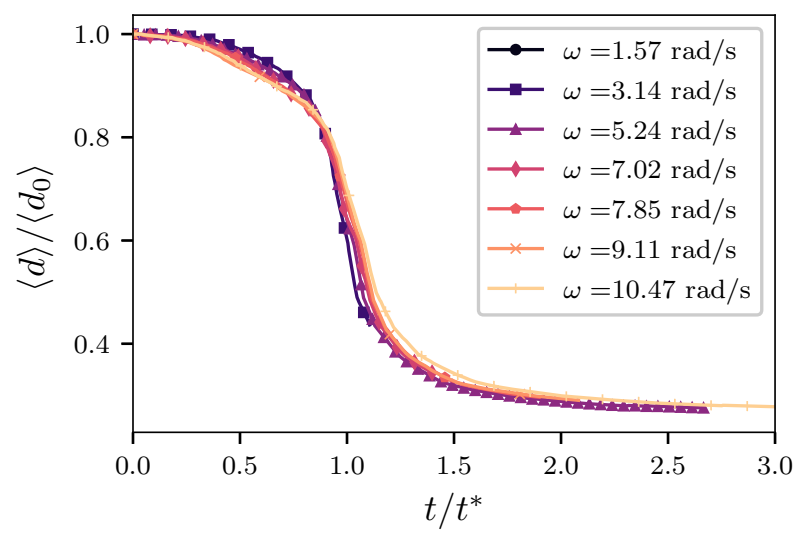

(b)

FIG. 11. The normalized specific surface $S / S_{0}$ (a), and normalized mean particle size (b) as a function of time normalized by the characteristic time $t^{*}$ for drums rotating at different speeds $\omega$.

place mostly at the toe of the flow, near to free surface, where the particles ejected from the shoulder impact the bed surface or roll down the steepest descent. We see that, although the probability is higher at the toe of the free surface, many breakage events occur also in the bulk of the flow as $\omega$ is increased. In all cases, the volume involved in particle breakage is small compared to the overall volume of the granular material. Part of the breakage events may be due to impacts in fluidized zones, but particles can also break inside the flow by shearing.

To get a better idea of the texture of granular flow, it is also interesting to map the local particle connectivity. Figure 13 shows in grayscale the number of contacts per particle. We see that the flow is globally less connected when $\omega$ increases. Moreover, the particles are much less connected inside the flowing layer as a result of inertial effects. This map makes clearly appear the borderline between the active and passive layers. We also observe that the volume of the active layer increases with $\omega$. 


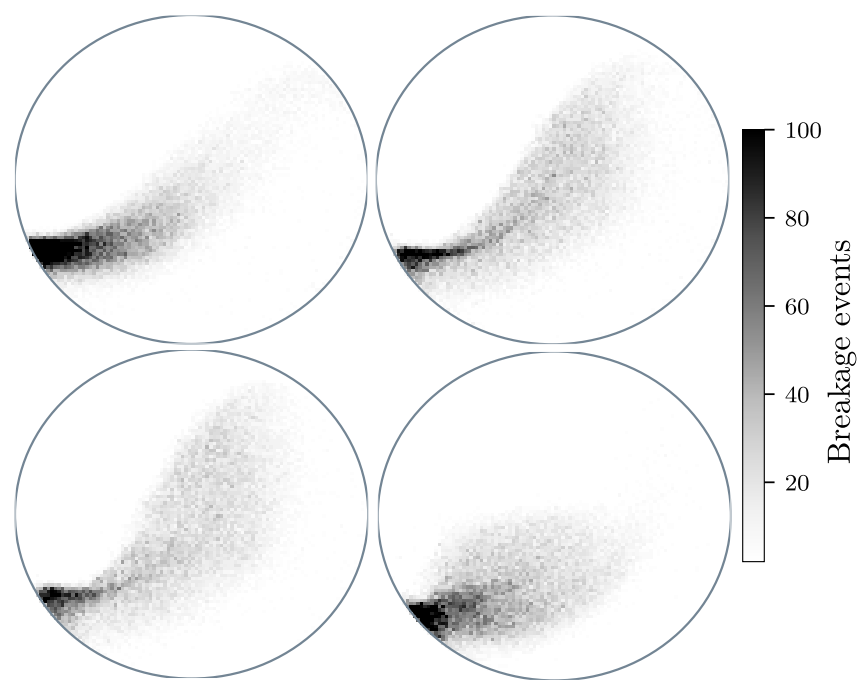

FIG. 12. Maps of local densities of breakage events during the whole simulation for $\omega=5.24,7.85,9.11$ and $10.47 \mathrm{rad} / \mathrm{s}$.
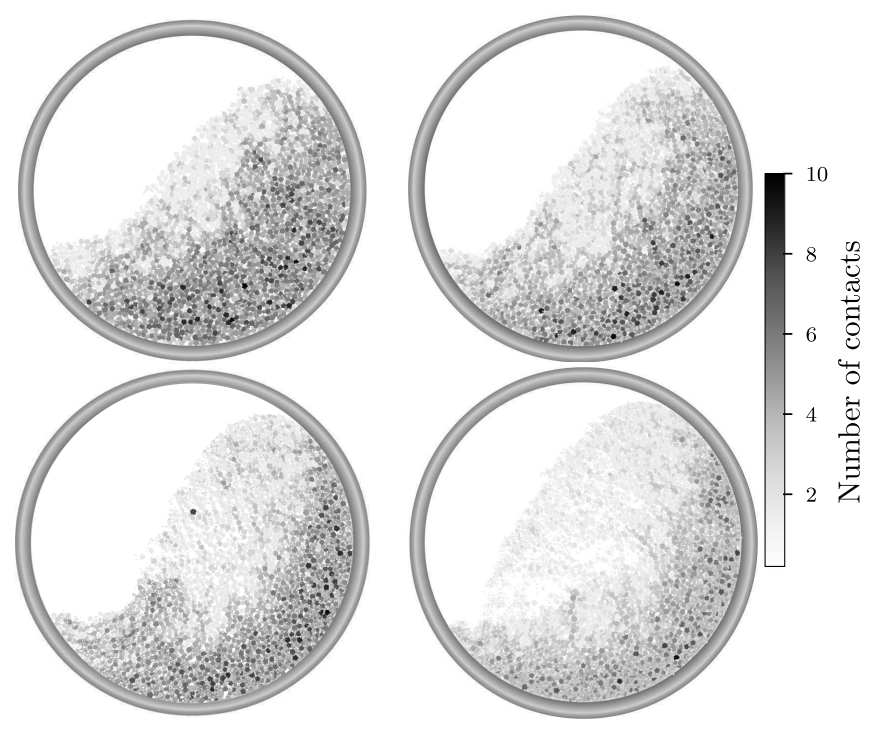

FIG. 13. Maps of particle connectivity after 13.75 rotations for $\omega=5.24,7.85,9.11$ and $10.47 \mathrm{rad} / \mathrm{s}$. This snapshot corresponds to the instants $0.73 t^{*}, 0.9 t^{*}, 0.96 t^{*}$ and $1.16 t^{*}$, respectively. The particle gray level is proportional to the number of contacts of the particle.

\section{B. Filling degree}

We now consider drums for the same rotation speed $\omega=$ $5.24 \mathrm{rad} / \mathrm{s}$ and size $R=0.075 \mathrm{~m}$, but filled at different levels $f=h_{0} / R$. The evolution of the mean particle size and specific surface are shown in Figs. 14(a) and 14(b). We observe here the same features as in the last subsection for all values of $f$. Figure 16(a) shows the grinding rate $\dot{S} / S_{0}$ as a function of $f$. Except for $f=20 \%$ and $f=25 \%$, the grinding rate declines as $f$ increases. The flow at low filling rates is in the 'sliding' regime: As the drum begins to rotate, the granular bed is sheared, but at the same time its center of mass swings back and forth along the drum wall around a mean position. This regime was also identified by Chou et al. [38] for filling degrees below $f=25 \%$. We observe this initial swinging of the bed, albeit to a much lesser extent, also at higher filling degrees. At low filling degrees, the swinging behavior continues during several drum rotations at the expense of reduced shearing of the bed and thus reduced breakage of particles. Lower shearing leads also to a reduced curved free surface. Figure 15 displays the free surface profiles for all the simulated filling degrees. The profiles are similar for all values of $f$ except for $f=20 \%$ and $f=25 \%$. In the following, we consider only the filling degrees above $25 \%$.

The characteristic time $t^{*}$, defined by Eq. (4), is a linear function of $f$ as shown in Fig. 16(b). In Fig. 17, the evolution of the mean particle size and specific surface are plotted against time normalized by $t^{*}$. Hence, for all filling degrees above $f=25 \%$, the only effect of the filling degree is to increase the characteristic time. No other feature of the grinding process seems to depend in any way on the filling degree.

\section{Drum size}

To examine the effect of drum size $R$ on the grinding process, we simulated drums of five different sizes. We performed two different sets of simulations. In the first set, $\omega$ was kept equal to $3.7 \mathrm{rad} / \mathrm{s}$ whereas in the second set the Froude number [Eq. (3)] was kept at 0.21. Figures 18 and 19 show the mean particle size and specific surface, respectively, as a function time for the two sets of simulations. The same features are observed as before, and we see that the grinding is increasingly faster when the drum size is increased for both sets of simulations. At constant Froude number, the time series of $\langle d\rangle$ and $S$ are quite close for different values of $R$. With a constant value of $\omega$ the evolution is much more dependent on $R$.

Figure 20(a) shows the grinding rate $\dot{S} / S_{0}$ as a function of drum size for the two sets of simulations. The behavior is well fit by linear functions. The slope is higher for constant $\omega$ as compared to the case of constant Froude number. This shows that the Froude number is a good scaling parameter as far as the drum size and rotation speed are involved. But for different filling degrees and particle sizes this scaling fails as clearly shown by the results of the previous subsection. The evolution of the characteristic time $t^{*}$ is shown in Fig. 20(b). It declines with increasing drum size in both cases. If we plot all the data as a function of time normalized by $t^{*}$, then they will naturally collapse (not shown here), as seen in the previous subsections.

\section{Particle shape}

Since in all the simulations for the scaling of particle breakage with system parameters we used pentagons, it is important to evaluate the influence of particle shape on the 


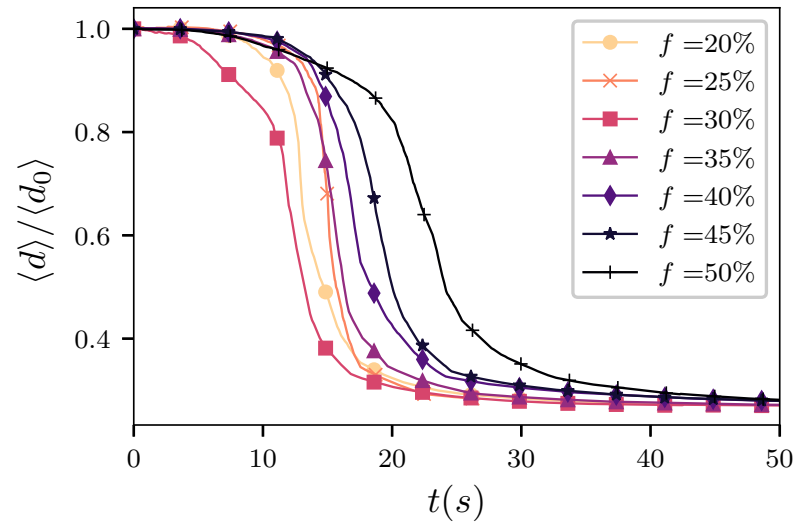

(a)

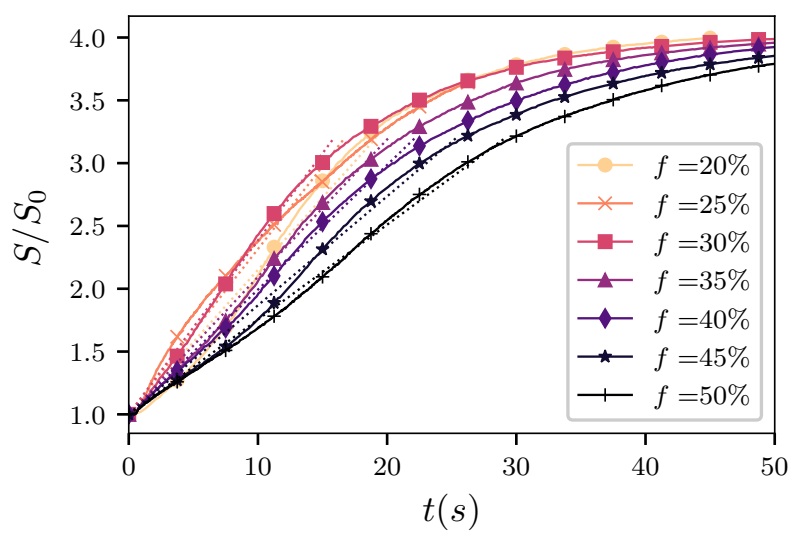

(b)

FIG. 14. (a) Mean particle size measured as a function of time for different values of the filling degree $f$ for the same drum size $R=$ $0.075 \mathrm{~m}$ and rotation speed $\omega=5.24 \mathrm{rad} / \mathrm{s}$. (b) Normalized specific surface $S / S_{0}$ as a function of time. The dashed lines are linear fits up to the transition point.

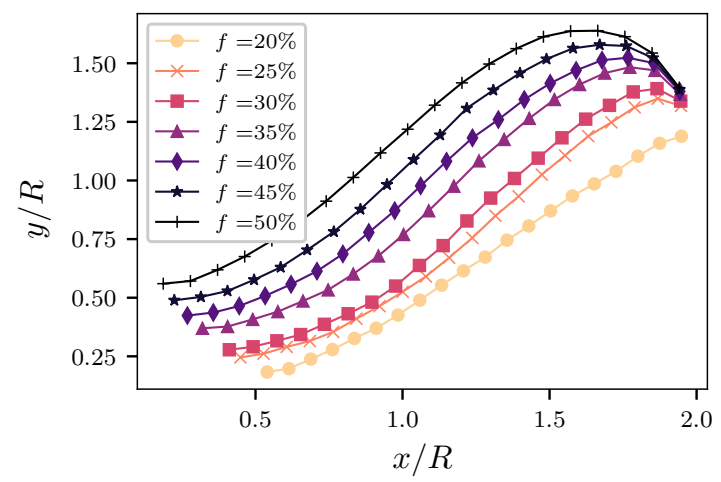

FIG. 15. Free surface profiles for different filling degrees.

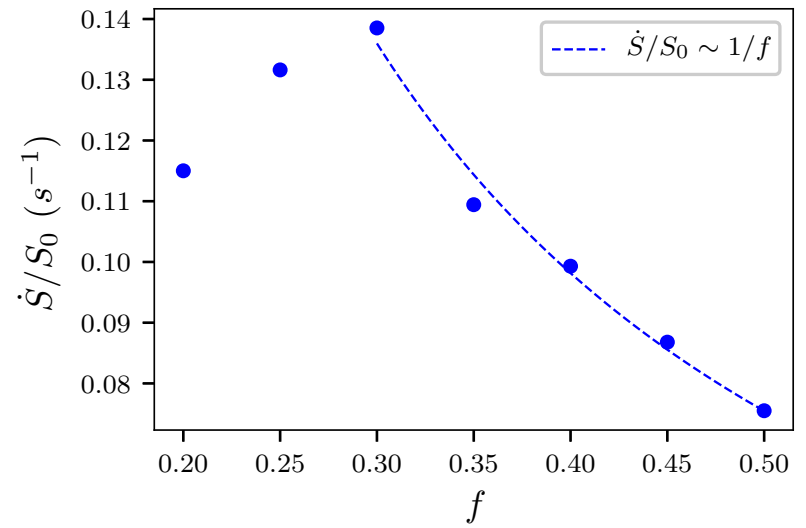

(a)

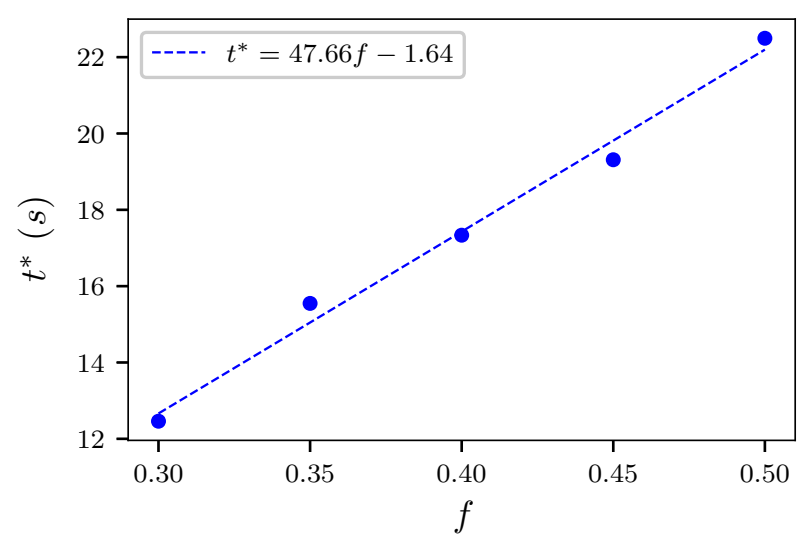

(b)

FIG. 16. (a) Rate of increase of specific surface as a function of the filling degree $f$. (b) Characteristic time $t^{*}$ as a function of $f$.

time evolution of specific surface and mean particle size for a few values of system parameters. Figure 1 displays examples of samples composed of regular polygons with increasing number of sides $n_{\text {sides }}$. In addition to $n_{\text {sides }}=5$, which is the reference shape used in all our simulations, we carried out simulations for $n_{\text {sides }}=6,7,9$, and 12, in a drum of size $R=0.075 \mathrm{~m}$, with rotation speed $\omega=5.24 \mathrm{rad} / \mathrm{s}$ and filling degree $f=0.51$. Figure 21 shows both the mean particle size and specific surface as a function of time for these different shapes. We see that the time series are similar, and the grinding rate before transition to the nonlinear regime quite weakly depends on the number of sides. The slightly higher grinding rate of rounder particles means that they are subject to higher force fluctuations or shear stresses. Higher forces can be a consequence of the fact that rounder particles can more easily roll down the free surface and therefore they acquire larger impact energies. 


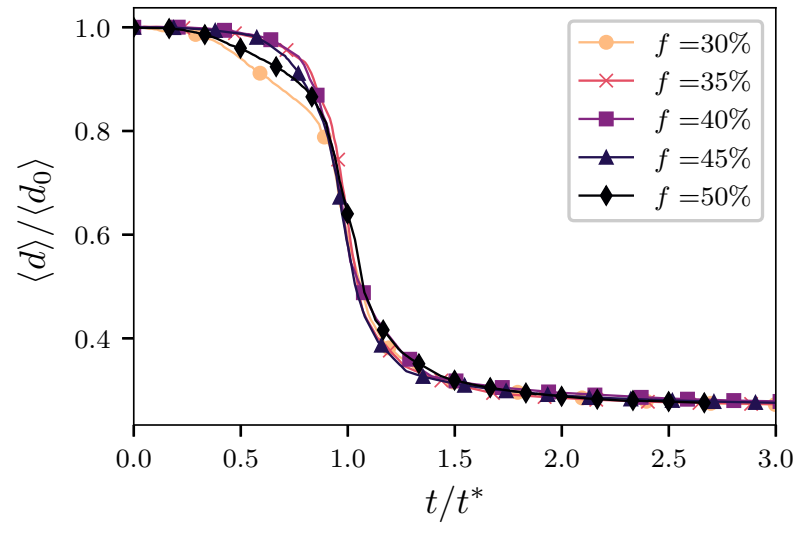

(a)

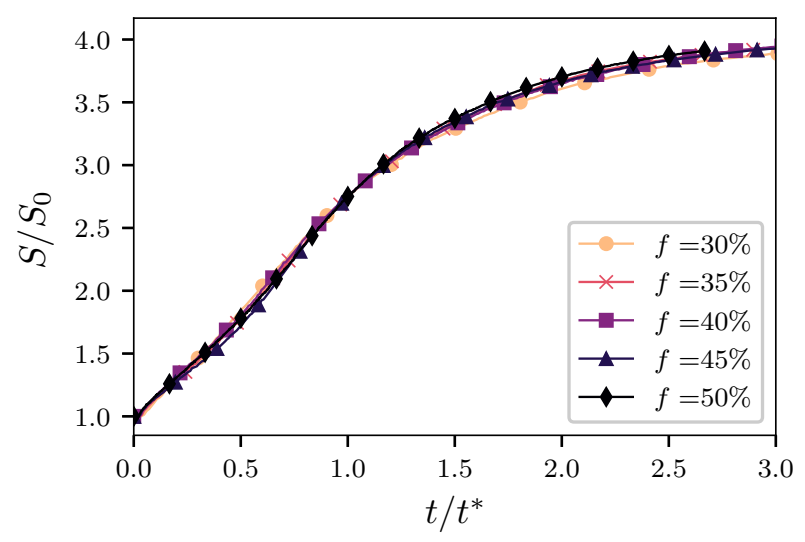

(b)

FIG. 17. (a) The normalized mean particle size $\langle d\rangle / d_{0}$ and (b) the normalized specific surface $S / S_{0}$, as a function of normalized time for different filling degrees.

\section{GENERAL SCALING LAW}

The partial parametric studies reported in the previous section suggest a power-law dependence of the grinding rate with respect to nearly all system parameters. We may thus look for a general scaling parameter $\Gamma$ combining all system parameters such that the grinding rate $\dot{S} / S_{0}$ would be an unique function of $\Gamma$. Alternatively, this scaling may be expressed in terms of the characteristic time $t^{*}$ as a function of $\Gamma$. The parameter $\Gamma$ may be defined as a general function of $\omega$, $R, d_{0}, h_{0}, \rho$, and $g$. However, to define a physically meaningful parameter, it is more convenient to work with dimensionless parameters that reflect the competing effects of various system parameters. The relevant dimensionless parameters are $\mathrm{Fr}=$ $R \omega^{2} / g$ (centrifugal force versus gravity), $R / d_{0}$ (finite size effect), and $f=h_{0} / R$ (filling degree). Although we did not change the internal cohesion $C_{n}$ of the particles, it is obvious that its value compared to the static stress induced by particle weights is an important factor for particle breakage

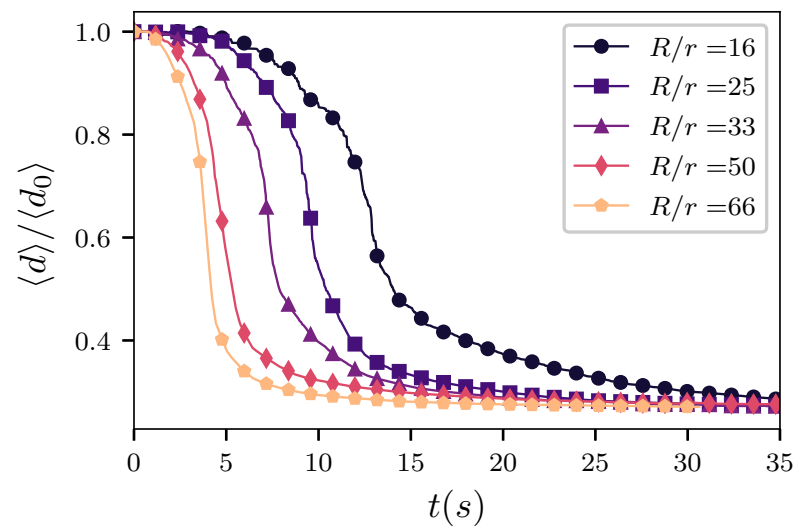

(a)

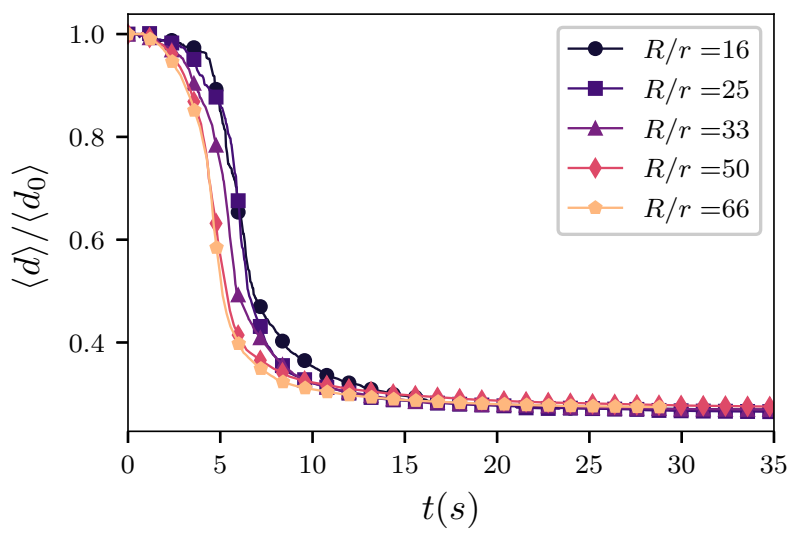

(b)

FIG. 18. Normalized mean particle size $\langle d\rangle / d_{0}$ as a function of time for drums of different sizes $R / r$ for a constant value of $\omega$ (a) and for a constant value of the Froude number (b).

rate. The mass $m$ is proportional to $\rho d_{0}^{2}$ and the static stress due to the weight of a single particle is $m / d_{0} \sim \rho g d_{0}$ (in two dimensions). Hence, the dimensionless number contributing to breakage is $\rho g d / C_{n}$.

We thus consider a scaling parameter of the following form:

$$
\Gamma=\operatorname{Fr}^{\alpha} f^{\beta}\left(\frac{R}{d_{0}}\right)^{\gamma}\left(\frac{\rho g d_{0}}{C_{n}}\right)^{\zeta} .
$$

We must determine the four exponents $\alpha, \beta, \gamma$, and $\zeta$ so that the values of the grinding rate $\dot{S} / S_{0}$ for all simulations collapse on a master curve as a function of $\Gamma$. Obviously, if for these values of the exponents $\Gamma$ is the scaling parameter for the grinding rate, then any function of $\Gamma$, including $\Gamma^{p}$ for arbitrary $p$ is also a scaling parameter. This means that only the ratios of the exponents $\alpha, \beta, \gamma$, and $\zeta$ are relevant. Hence, in practice we have three exponents to fix.

If we use the values of the exponents evidenced by the partial parametric studies of the last section for the grinding rate, then we may obtain a linear dependence between the 


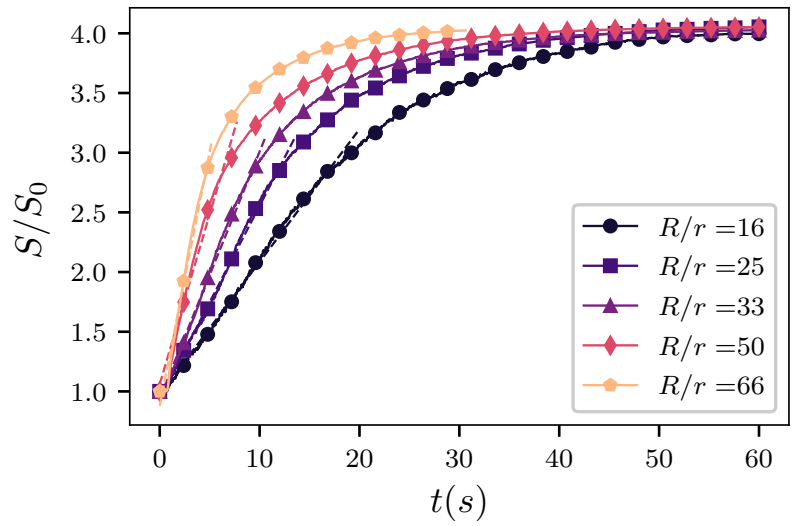

(a)

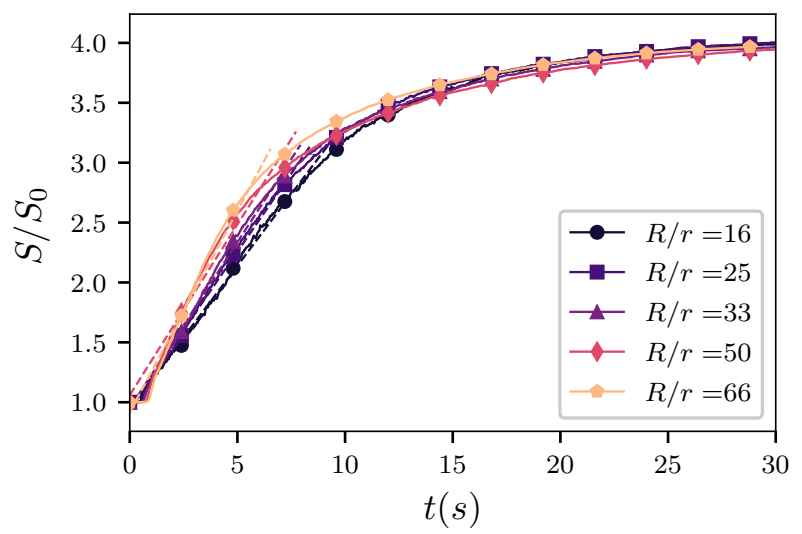

(b)

FIG. 19. Normalized specific surface $S / S_{0}$ for drums of different sizes $R / r$ for a constant value of $\omega$ (a) and for a constant value of the Froude number (b). The dashed lines are linear fits below the transition point.

latter and $\Gamma$. For rotation speed $\omega$, a dependence $\dot{S} / S_{0} \sim \omega^{3 / 2}$ [see Fig. 10(a)] was observed. We therefore set $\alpha=3 / 4$. For the filling degree $f$, we have $\dot{S} / S_{0} \sim 1 / f$ [by judging from the behavior of $t^{*}$ in Fig. 16(a)] so that we may set $\beta \simeq-1$. For the drum size $R / r$, according to Fig. 20(a), we have $\dot{S} / S_{0} \sim R / d_{0}$ at a constant value of $\omega$. By accounting for the values of $\alpha$ and $\beta$, given that $f=h_{0} / R$ and Froude number is linear in $R$, we should set $\gamma \simeq 1 / 4$. Finally, to determine $\zeta$, we use two sets of simulations with the two values of $d_{0}$, and choose the value of $\zeta$ in such a way to make the grinding rates collapse as a function of $\Gamma$. This procedure yields $\zeta \simeq 3 / 2$.

As $\Gamma$ is a dimensionless parameter, we need a timescale to transform also the grinding rate, which has the inverse time dimension, to a dimensionless parameter. This time can not be $t^{*}$, which is defined from the grinding rate. We have three different times in the system: $\omega^{-1}$ (driving time), $\left(d_{0} / g\right)^{1 / 2}$ (rearrangement time due to gravity), and $\left(C_{n} / \rho\right)^{1 / 2} / g$. We may refer to the latter as 'breaking time'. A particle should gain

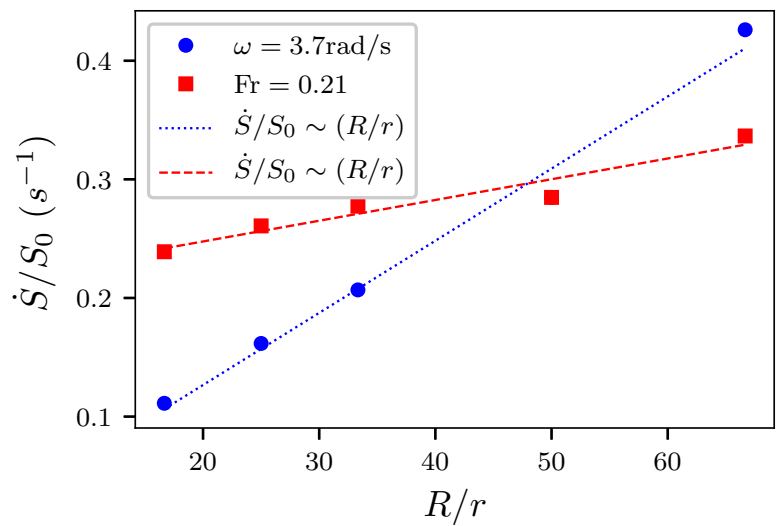

(a)

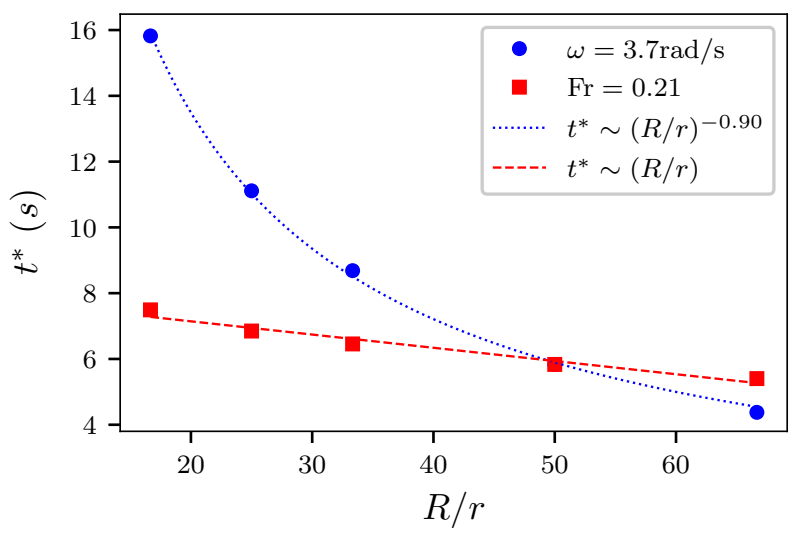

(b)

FIG. 20. (a) The rate of increase of the normalized specific surface shown in Fig. 19. (b) Characteristic time as a function of drum size ratio $R / r$ for the two sets of simulations. The dashed lines are power-law fits to the data.

enough kinetic energy between two impact events to be able to exert a stress larger than $C_{n}$ for particle breakage. If $\tau$ is the typical time between two events, the the order of magnitude of the velocity gained by a particle is $g \tau$. The corresponding energy per unit volume is $\sim \rho(g \tau)^{2}$. Equaling this energy with $C_{n}$, we get

$$
\tau=\frac{1}{g}\left(\frac{C_{n}}{\rho}\right)^{1 / 2}
$$

The only timescale that does not interfere with the selected values of the aforementioned exponents is $\tau$. For this reason, we consider below the dimensionless grinding rate $\tau \dot{S} / S_{0}$ and its scaling with $\Gamma$.

Figure 22 displays the dimensionless grinding rate as a function of $\Gamma$ for all our simulations. Remarkably, all the data points collapse on a linear function,

$$
\frac{\tau \dot{S}}{S_{0}} \simeq 37.73 \Gamma=37.73 \frac{1}{f}\left(\frac{R \omega^{2}}{g}\right)^{3 / 4}\left(\frac{R}{d_{0}}\right)^{1 / 4}\left(\frac{\rho g d_{0}}{C_{n}}\right)^{3 / 2} .
$$




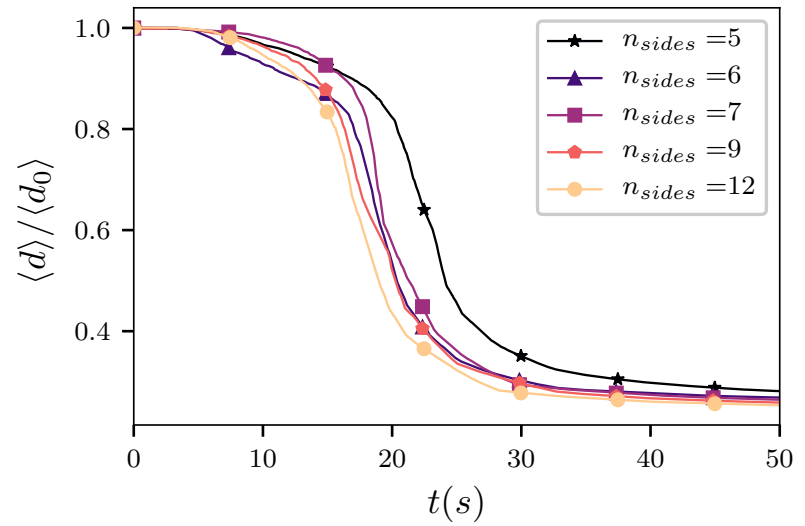

(a)

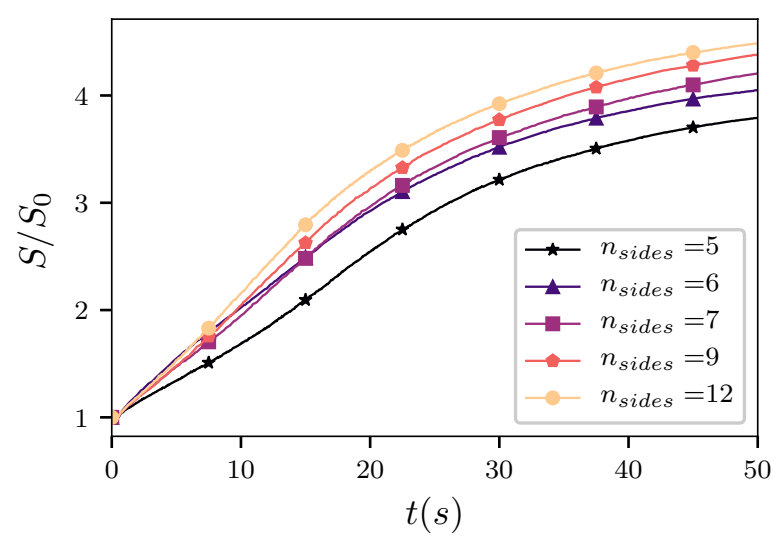

(b)

FIG. 21. The normalized mean particle size $\langle d\rangle / d_{0}$ (a) and normalized specific surface $S / S_{0}$ (b) as a function of time in drum flows composed of regular polygons of different numbers of sides for fixed drum size, rotation speed and filling degree.

This scaling involves all control parameters and material parameters of the system. It predicts the dependence of the grinding rate with respect to parameters such as $C_{n}, g$, and $\rho$, which were not varied in this work. The validity of this scaling can thus be easily tested by performing further simulations with different values of these parameters. Note that the dependence on particle size $d_{0}$ in the above expression is not a finite size effect. The grinding rate increases as $\tau \dot{S} / S_{0} \propto d_{0}^{5 / 4} g^{3 / 4}$. In combination with $\rho$, this can be written as $m\left(d_{0} / g\right)^{-3 / 4}$ where $m=\rho d_{0}^{2}$. This means that $d_{0}$ is involved through both the mass of the particles and microscopic time $\left(d_{0} / g\right)^{1 / 2}$ although in the initial search of the scaling parameter we used the ratio $R / d_{0}$, which is a finite size factor. It should also be noted that the decrease of grinding rate $\dot{S} / S_{0}$ with increasing filling degree does not mean that the absolute rate of grinding $\dot{S}$ decreases since $S_{0}$ increases in 2D as $h_{0}^{3 / 2}$, implying $\dot{S} \sim f^{1 / 2}$.

Equivalently, from Eq. (7) we get the characteristic time $t^{*}$

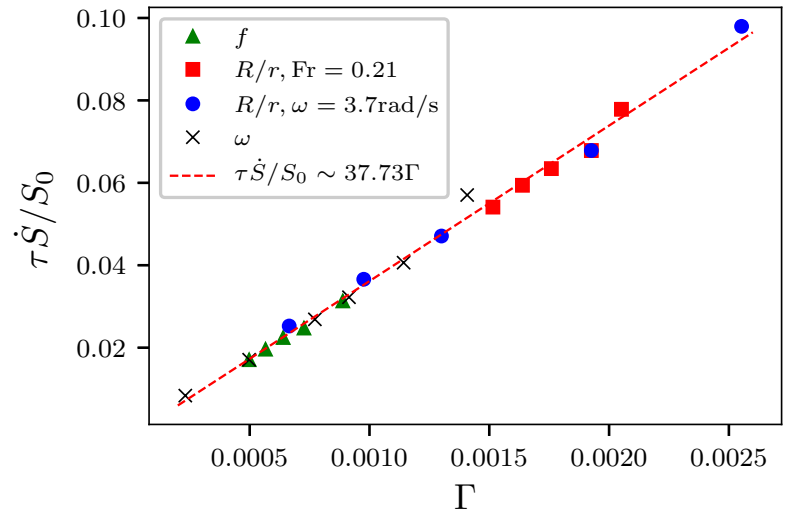

FIG. 22. Dimensionless grinding rate as a function of the scaling parameter $\Gamma$ in Eq. (5) with $\alpha=3 / 4, \beta=-1, \gamma=1 / 4$, and $\zeta=3 / 2$ for all our simulations with different values of system parameters. The symbols refer to different sets of simulations in which every time a single parameter (filling degree $f$, rotation speed $\omega, R / r$ at constant Froude number or constant rotation speed) is varied.

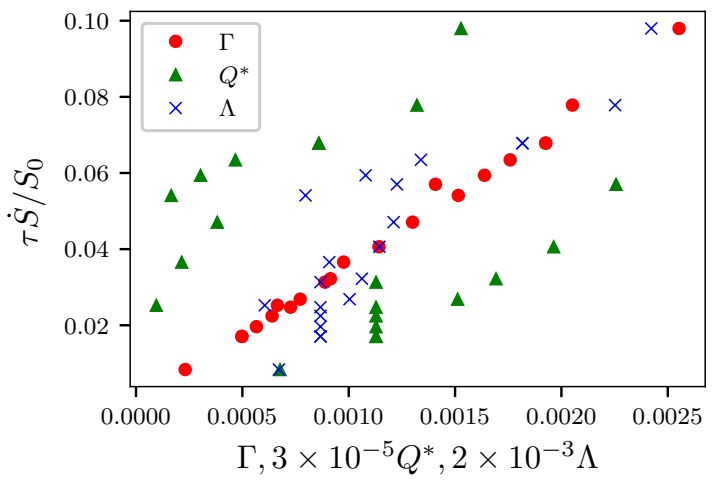

FIG. 23. Comparison between the proposed scaling law in Eq. (5) and the same data plotted as function of $\Lambda$ [39] and $Q^{*}$ [40]. A prefactor was applied to bring the data to the same range.

as a function of system parameters:

$$
\frac{t^{*}}{\tau} \simeq 0.073\left(\frac{R \omega^{2}}{g}\right)^{-\frac{3}{4}}\left(\frac{R}{d_{0}}\right)^{-\frac{1}{4}}\left(\frac{\rho g d_{0}}{C_{n}}\right)^{-\frac{3}{2}}\left(\frac{h_{0}}{R}\right)
$$

The characteristic time decreases with increasing $R, \omega, g, \rho$, and $d_{0}$, and it increases with increasing $C_{n}$ and $h_{0}$.

Several authors have proposed scaling parameters for rotating drums. For example, Taberlet et al. [39] proposed the parameter

$$
\Lambda=\left(\operatorname{Fr} \frac{d}{R}\right)^{1 / 4} \frac{R}{W},
$$

which involves $\mathrm{Fr}, R / d$, and $R / W$, where $W$ is the width of the drum in 3D. Pignatel et al. [40] introduced the parameter

$$
Q^{*}=\frac{1}{2} \operatorname{Fr}^{1 / 2}\left(\frac{R}{d}\right)^{3 / 2} \text {. }
$$


Although $\Lambda$ and $Q^{*}$ were introduced for granular flow rather than particle breakage, it is interesting to see how these parameters scale our data. In Fig. 23 we have plotted the dimensionless grinding rate as a function of $\Gamma, \Lambda$ (by setting $W=1$ ) and $Q^{*}$ by multiplying $\Lambda$ and $Q^{*}$ by a scale factor to bring all the points to the range of values of $\Gamma$. We see that our data points as a function of both $\Lambda$ and $Q^{*}$ are widely scattered while they collapse for $\Gamma$.

\section{CONCLUSIONS}

In this paper, we analyzed the evolution of particle breakage in a $2 \mathrm{D}$ rotating drum for a broad range of values of rotation speed, drum size, filling degree, and particle size and shape. Each particle can break down to an unbreakable primary volume as a constant fraction of the volume of the mother particle. We were interested in the influence of each system parameter on the evolution of specific surface and mean particle size. The specific surface increases almost linearly with time up to a transition point to a nonlinear regime where many unbreakable fragments are generated, and thus the probability of breakage declines. For all values of system parameters, this point corresponds to the same amount of specific surface equal to slightly more than half the maximum specific surface that can be generated in the simulations. This point was used to define a characteristic time.

When time is normalized by the characteristic time, all the data points collapse on the same master curve. By analyzing the dependence of this time or the grinding rate on the system parameters, we arrived at a scaling parameter incorporating all system parameters. This parameter has nontrivial exponents, and it implies an increase of the breakage rate with increasing rotation speed, drum size, particle size and density, and a decrease of breakage rate with increasing filling degree and internal cohesion of the particles. This scaling is a result of the combined effects of multicontact mechanical interactions inside the flow, granular flow regimes in a rotating drum with their geometrical features, and operating parameters.

The scaling parameter is dimensionless and fully constrained by all the available dimensional parameters of the system. For this reason, it would be interesting to further check its predictions of the grinding rate by means of simulations with modified particle density, particle strength, and gravity. Another line of research is the correlation between the scaling parameter and granular flow variables such as the shape of the free surface and slip at the walls. Indeed, we previously characterized the flow of unbreakable particles in a rotating drum in 3D where a single scaling parameter was found to describe the flow variables. However, the scaling of particle breakage, as evidenced in this work, is very different from that parameter. This difference indicates that, besides flow variables such as free surface shape, particle breakage depends on the flow patterns inside the drum. For example, intense breakage of particles may occur in a small volume of the drum located at the toe of the cascading flow. But due to lower volume involved, it does not lead to a globally higher breakage rate. This aspect regarding local breakage probabilities merits further work in the future.

Another important aspect of particle breakage is size segregation while smaller fragments are generated. We did not perform a quantitative investigation of this effect because of its complexity, deserving a minute analysis. This complexity involves the timescales of the particle breakage and segregation processes. The breakage events are localized in some parts of the drum depending on the values of the parameters, as shown in Fig. 12, but the flow tends to spread the generated fragments throughout the granular bed, and thus we observe a nearly homogeneous distribution of damaged particles as shown in Fig. 6. This is a continuous process in which because of continuous change of particle sizes no clear pattern of segregation emerges. Of course, this does not prove that there is no size segregation at all. For example, we observe that larger fragments are mostly found at the drum wall or at the free surface in the last two snapshots of Fig. 6. In general, particle breakage seems to enhance mixing rather than segregation. However, more work is needed to quantify such effects for the range of system parameters investigated in this paper.

Finally, in this work the influence of wall friction and roughness was not considered. We believe that the sliding at the drum wall can be partially eliminated by wall roughness, as often used in industrial applications. Note that the sliding at the walls can also occur as a thin shear band even in the presence of rough walls. This point needs to be investigated for the whole range of parameters considered in this work.
[1] S. M. Iveson, J. D. Litster, K. Hapgood, and B. J. Ennis, Powder Technology 117, 3 (2001).

[2] Ristow G.H., "Mixing and segregation in rotating drums," (Springer, Dordrecht, 2000) pp. 311-320.

[3] S. H. Chien, G. Carmona, L. I. Prochnow, and E. R. Austin, J Environ Qual 32, 1911 (2003).

[4] A. Barkouti, E. Rondet, M. Delalonde, and T. Ruiz, Journal of Food Engineering 111, 234 (2012).

[5] A. Nosrati, J. Addai-Mensah, and D. J. Robinson, Hydrometallurgy 125-126, 90 (2012).

[6] C. Mayer-Laigle, R. K. Rajaonarivony, N. Blanc, and
X. Rouau, Bioengineering 5, 50 (2018).

[7] A. S. Erdem and S. L. Ergün, Minerals Engineering 22, 660 (2009).

[8] T. Iwasaki, T. Yabuuchi, H. Nakagawa, and S. Watano, Advanced Powder Technology 21, 623 (2010).

[9] V. K. Gupta, Powder Technology 305, 714 (2017).

[10] D.-H. Nguyen, E. Azéma, F. Radjai, and P. Sornay, Physical Review E 90, 012202 (2014).

[11] Cegeo, B. Saint-Cyr, K. Szarf, C. Voivret, E. Azéma, V. Richefeu, J.-Y. Delenne, G.Combe, C. Nouguier-Lehon, P. Villard, P. Sornay, M. Chaze, and F. Radjai, Eur. Phys. Letter 
, 5 (2012).

[12] Powder Technology 143-144, 144 (2004).

[13] M. J. Metzger and B. J. Glasser, Powder Technology 237, 286 (2013).

[14] M. Marigo and E. H. Stitt, KONA Powder and Particle Journal 32, 236 (2015).

[15] J. Herbst and D. Fuerstenau, International Journal of Mineral Processing 7, 1 (1980).

[16] V. K. Gupta and S. Sharma, Advanced Powder Technology 25, 625 (2014).

[17] R. M. De Carvalho and L. M. Tavares, Minerals Engineering 43-44, 91 (2013).

[18] Powder Technology 323, 374 (2018).

[19] P. W. Cleary, R. D. Morrison, and G. W. Delaney, Minerals Engineering 128, 56 (2018).

[20] L. Vogel and W. Peukert, International Journal of Mineral Processing 74, 329 (2004).

[21] F. Shi and T. Kojovic, International Journal of Mineral Processing 82, 156 (2007).

[22] M. Capece, E. Bilgili, and R. Davé, Chemical Engineering Science 117, 318 (2014).

[23] B. Caicedo, M. Ocampo, and L. Vallejo, Computers and Geotechnics 80, 383 (2016).

[24] Y. L. Ding, R. N. Forster, J. P. Seville, and D. J. Parker, Chemical Engineering Science 56, 3737 (2001).

[25] M. Cacciuttolo and A. Arunakumari, Scale-Up Considerations for Biotechnology-Derived Products (CRC Press, 2005) pp. $129-160$.

[26] D.-H. Nguyen, E. Azéma, P. Sornay, and F. Radjai, Physical Review E 91, 022203 (2015).

[27] D. Cantor, E. Azéma, P. Sornay, and F. Radjai, Comput. Particle Mech. 4 (2017), 10.1007/s40571-016-0129-0.

[28] T-Trung. Vo, P. Mutabaruka, J-Y. Delenne, S. Nezamabadi, and F. Radjai, EPJ Web Conf. 140, 08021 (2017).

[29] L. F. Orozco, J. Y. Delenne, P. Sornay, and F. Radjai, Int. J. Solids Struct. 166, 47 (2019).

[30] L. Orozco, D. Nguyen, J.-Y. Delenne, P. Sornay, and F. Radjai, Powder Technology 362, 157 (2019).

[31] M. Jean, V. Acary, and Y. Monerie, Philosophical Transactions of the Royal Society of London A: Mathematical, Physical and Engineering Sciences 359, 2497 (2001).

[32] F. Radjai and V. Richefeu, Mechanics of Materials 41, 715 (2009).

[33] F. Radjai and F. Dubois, Discrete-element modeling of granular materials (Wiley-Iste, 2011).

[34] D.-H. Nguyen, E. Azéma, P. Sornay, and F. Radjai, Eur. Phys. J. E 41, 50 (2018).

[35] C. Voivret, F. Radjaï, J.-Y. Delenne, and M. S. E. Youssoufi, Physical Review E 76, 021301 (2007).

[36] P. Mutabaruka, M. Taiebat, Roland J.-M. Pellenq, and F. Radjai, Physical review E 100, 042906 (2019).

[37] J. Mellmann, Powder Technology 118, 251 (2001).

[38] H. T. Chou and C. F. Lee, Granular Matter 11, 13 (2009).

[39] N. Taberlet, P. Richard, and J. Hinch, Phys. Rev. E 73, 1 (2006).

[40] F. Pignatel, C. Asselin, L. Krieger, I. C. Christov, J. M. Ottino, and R. M. Lueptow, Physical Review E - Statistical, Nonlinear, and Soft Matter Physics 86, 1 (2012). 